\title{
Laparoscopic Thrombectomy of Renal Angiomyolipoma Level III
}

\author{
Cesar A. Britto, Hiram N. Paiva, Paulo J. de Medeiros, Pedro Sales L. de Carvalho, Filipe C.L.R. \\ de Medeiros, Thiago S. da Costa \\ Departamento of Urology, Onofre Lopes University Hospital, University of Rio Grande do Norte \\ (UFRN), Natal, Brazil
}

\begin{abstract}
Purpose: Renal angiomyolipoma (AML) is a benign tumor, corresponding to approximately 3\% of solid renal tumors, which has in its composition endothelial cells, myocytes and adipocytes. One of the rare complications of this type of tumor is linfonodal involvement and tumor venous dissemination forming thrombus in the renal vein and inferior vena cava and may even reach the right atrium. Surgical treatment of this type of tumor is performed mainly by open surgery, but this video shows the laparoscopic approach for level III of angiomyolipoma, showing that this approach is feasible and reproducible by any trained and experienced surgeon.

Materials and Methods: Woman of 65 years with back pain, ultrasound examination showed a tumor in the right kidney, and follow-up computed tomography revealed aspects of angiomyolipoma. We performed radical nephrectomy with thrombectomy by laparoscopy.

Results: The patient recovered well, enjoying all the benefits of laparoscopic surgery. There were no complications during surgery.

Conclusions: The surgical approach of laparoscopic renal angiomyolipoma is a perfectly feasible, bringing numerous benefits to the patient, and can establish itself as a breakthrough in treating this type of tumor.
\end{abstract}

Int Braz J Urol. 2011; 37 (Video \#5): 558-559

Available at: www.brazjurol.com.br/videos/july_august_2011/Britto_558_559video.htm

\author{
Correspondance address: \\ Dr. Cesar A. Britto \\ Rua Vereador João Alves da Silva Filho, 784 \\ Natal, RN, 59020-110, Brazil \\ Fax: + 5584 9413-7399 \\ E-mail: cesar_britto@yahoo.com
}




\section{EDITORIAL COMMENT}

The authors present a wonderful video demonstrating the laparoscopic removal of inferior vena cava (IVC) tumor thrombus associated with a right renal angiomyolipoma.

While the video demonstrates the technique of removing the thrombus, the experienced surgeon who manages these patients understands that there has been quite extensive laparoscopic dissection. The right colon and the small bowel (to include the duodenum) have been extensively mobilized. Significant vascular dissection and mobilization has been performed to include the left renal vein, the right renal hilum, the infrarenal vena cava and the suprarenal vena cava. I suspect multiple lumbar veins have been divided (as the field was bloodless after the venotomy was performed and the thrombus extracted). In addition, as this was a level III thrombus, multiple short hepatic veins communicating with the caudate lobe of the liver were dissected and divided. These are all critical steps towards completely and safely extracting vena cava tumor thrombus.
I applaud the authors for demonstrating this beautiful case. However, very few surgeons have the technical capabilities to do this procedure. They need to have tremendous experience with kidney cancers and IVC thrombus, and they should have significant laparoscopic experience.

This case is highly select. The non-malignant histology likely contributed to the ease of thrombus removal. A surgeon familiar with IVC thrombus (most always associated with kidney cancer) understands that many patients will have thrombus adherence to the caval wall. In some instances, the thrombus is removed in a piecemeal or in a fragmented fashion because of the same adherence characteristics. A laparoscopic approach might be an option for a few patients as in this case where the radiographic images suggest that the thrombus might be free floating and nonadhered. Otherwise, the cavotomy needs to be extended in a longitudinal fashion for complete exposure and removal of the thrombus.

Dr. Wade J. Sexton

Associate Member

Department of Genitourinary Oncology

Moffitt Cancer Center

Tampa, FL 33612, USA

E-mail: wade.sexton@Moffitt.org 